\title{
Réponse glycémique induite par quatre boissons alcoolisées locales du Bénin chez des sujets adultes jeunes sains
}

\author{
M. GOMINA ASSOUMANOU*, D. H. SAKA et S. A. AKPONA \\ UERS de Biochimie et de Biologie Moléculaire, Faculté de Médecine, Université de Parakou. \\ BP : 123 Parakou, Bénin. \\ *Auteur correspondant, E-mail : elboutraguero@yahoo.fr; Tél : + 22997107779
}

\section{RESUME}

La consommation d'éthanol inhibe la néoglucogenèse et peut théoriquement diminuer la réponse glycémique postprandiale. L'objectif de cette étude était d'explorer les effets de quatre boissons alcoolisées locales du Bénin consommées à jeun et avec du pain, sur la réponse glycémique chez des sujets adultes jeunes sains. Après avis éthique, 40 sujets ( 24 hommes, 16 femmes), volontaires, ont été sélectionnés parmi les étudiants de l'Université de Parakou (Bénin) et répartis en 4 groupes de 10. Chaque groupe de sujets a consommé $1000 \mathrm{KJ}$ de l'une des quatre boissons (tchoukoutou, sodabi, vin de palme frais, vin de palme fermenté) seule et accompagnée de $100 \mathrm{~g}$ de pain de blé. La réponse glycémique pendant 2 heures a été évaluée à partir des glycémies veineuses. A jeun, le vin de palme fermenté était la boisson qui a le plus réduit la glycémie postprandiale $(95,72 \%)$ tandis que consommées avec du pain, la plus grande réduction de la glycémie a été observée avec le vin de palme frais $(64,06 \%)$. Le tchoukoutou avait l'index glycémique le plus élevé (20,59\%) tandis que le vin de palme fermenté possédait la charge glycémique la plus élevée $(115,43 \%)$. Cette étude permet d'envisager l'utilisation de ces boissons alcoolisées locales dans la prise en charge diététique du diabète sucré.

() 2011 International Formulae Group. All rights reserved.

Mots clés : glycémie, tchoukoutou, sodabi, vin de palme, index glycémique.

\section{INTRODUCTION}

La consommation d'alcool sous diverses formes est l'un des fléaux ubiquitaires depuis des temps immémoriaux. La production des boissons alcoolisées peut être artisanale ou industrielle. Si la production industrielle se fait par un procédé plus élaboré, celle artisanale de boissons alcoolisées en Afrique sub-saharienne relève de procédés traditionnels presque identiques d'un pays à l'autre (Noll, 2008; Adeleke et Abiodun, 2010). La composition de ces boissons de fabrication artisanale est variable selon le type de boisson (Obiora et al., 2007 ; Adeleke et Abiodun, 2010). La consommation de boissons alcoolisées locales s'est accrue de nos jours en Afrique noire en raison de leur caractère socioculturel et de leur coût relativement bas par rapport aux boissons alcoolisées de fabrication industrielle et importées.

Durant ces dernières années, il a été clairement démontré que les boissons alcoolisées ont des effets différents sur la 
réponse glycémique et insulinique post prandiale (Brand-Miller et al., 2007). En dehors de l'action hypoglycémiante connue de l'éthanol (Verdy et Diallo, 1968 ; Sporer et al., 1992), cette variabilité de la réponse glycémique après ingestion d'une boisson alcoolisée dépend de sa composition. En effet, les flavonoïdes contenus dans le vin diminuent la réponse glycémique en augmentant la réponse insulinique (Anderson et Polansky, 2002 ; Bryans et al., 2007). De même, les acides organiques qui peuvent être produits au cours du processus de la fermentation des boissons alcoolisées réduisent la réponse glycémique post prandiale en accélérant la vidange gastrique (Hunt et Knox, 1972) et en inhibant l'action de l' $\alpha$-amylase (Todesco et al., 1991 ; Liljeberg et al., 1995), ce qui aboutit à une diminution de la quantité d'ose libre pour l'absorption intestinale. En outre, les acides aminés d'origine végétale, en particulier la leucine, sont hypoglycémiants en raison de leur caractère insulinotrope (Layman, 2003). La réponse glycémique induite par les boissons alcoolisées (bière, vin, spiritueux) produites dans les pays industrialisés chez des sujets adultes jeunes sains est connue (BrandMiller et al., 2007). Qu'en est-il des boissons alcoolisées de fabrication locale couramment consommées au Bénin?

L'objectif de cette étude était d'explorer les effets de quatre boissons alcoolisées locales du Bénin consommées à jeun et avec du pain, sur la réponse glycémique chez des sujets béninois adultes jeunes et sains.

\section{MATERIEL ET METHODES}

\section{Matériel}

\section{Réactifs et appareils utilisés}

Les réactifs utilisés dans le cadre de ce travail étaient: des kits pour le dosage de la glycémie (Glucose MR Enzymatic colorimetric method ENDPOINT du laboratoire Linear Chemicals référence 1129010); des kits pour le dosage des transaminases ASAT et ALAT MR Enzymatic colorimetric method ENDPOINT du laboratoire Linear Chemicals références 1109000 et 1105000 respectivement; des kits pour le dosage de l'urée et créatinine plasmatique de marque ILO Diagnosctics références URUV-3400 et CRCO-5705 respectivement.

Autre matériel utilisé dans ce travail était constitué d'un spectrophotomètre à aspiration de marque Microlab 300, d'une centrifugeuse de marque Sivma, d'un bainmarie de marque Selecta et d'un alcoomètre avec thermomètre de marque Assistent.

\section{Boissons testées}

Les boissons testées dans cette étude étaient: le tchoukoutou (bière de mil locale) titré à $3 \%$ d'alcool, le sodabi titré à $40 \%$ d'alcool, le vin de palme frais titré à $3 \%$ d'alcool et le vin de palme fermenté titré à 8\% d'alcool (Chevassus et Favier, 1976; Kayode et al., 2007 ; Adeleke et Abiodun, 2010). Le Tableau 1 montre la composition physicochimique pour 100 millilitres de chaque boisson utilisée.

\section{Aliment standard utilisé}

L'aliment standard utilisé était du pain de blé. Nous avons choisi 100 grammes de pain de blé (contenant 50 grammes de glucides) tartinés de margarine (acquise de Vital Pharmaceutical Ltd. London-United Kingdom) afin d'obtenir une référence pour un index glycémique à $100 \%$.

\section{Population d'étude}

Les sujets de cette étude ont été sélectionnés après un consentement éclairé, lu et approuvé. Il s'agissait d'étudiants en médecine de l'Université de Parakou, des deux sexes, ayant une prise occasionnelle de boissons alcoolisées. Ont été inclus dans cette étude, les sujets adultes jeunes volontaires (18-27 ans), ne présentant aucune entéropathie exsudative et ayant un bilan biologique de base (glycémie à jeun, urée sanguine, créatinine plasmatique, ALAT et ASAT) avec des constantes physiques (indice de masse corporelle, pression artérielle) normaux. Les 
paramètres des sujets sélectionnés sont présentés dans le Tableau 2. Ont été non inclus, les femmes enceintes ou allaitantes, les sujets diabétiques ou avec un antécédent familial de diabète sucré, les sujets souffrant d'une hépatopathie quelconque, d'une pancréatopathie, les sujets présentant des troubles de la crasse sanguine, les sujets ayant un antécédent d'ulcère gastroduodénal ou de gastrite, les sujets sous traitement médicamenteux quelconque et les sujets n'ayant pas donné leur consentement éclairé. Ces critères nous ont permis de sélectionner au total 40 sujets des deux sexes (24 hommes, 16 femmes). Selon l'utilisation du modèle humain aux fins d'une expérimentation basée sur les critères de l'OMS/FAO pour la réalisation d'une étude sur les réponses glycémiques (FAO/WHO, 1998), les sujets sélectionnés ont été répartis en quatre (4) groupes de dix (10): un premier groupe composé de sept (7) hommes et de trois (3) femmes pour l'étude 1 ; un deuxième groupe composé de sept (7) femmes et de trois (3) hommes pour l'étude 2 ; un troisième groupe composé de huit (8) hommes et deux (2) femmes pour l'étude 3 ; un quatrième groupe composé de six (6) hommes et quatre (4) femmes pour l'étude 4 .

\section{Méthodes}

Nous avons réalisé une étude expérimentale, qui a couvert la période du 02 janvier 2010 au 12 octobre 2010 après approbation du protocole par le Comité National Provisoire d'Ethique de la Recherche en Santé (CNPERS) du Bénin.

\section{Procédure de l'expérimentation}

Les sujets sélectionnés selon les critères sus cités ont été mis à jeun 10 à 12 heures avant l'expérimentation et sevrés de toute consommation d'alcool la veille. Chacun des 40 sujets a été reçu le jour de l'expérimentation dans la salle préparée à cet effet le matin à 8 heures. Après 10 à 15 minutes de repos, un cathéter veineux a été mis en place dans une des veines cubitales pour recueillir un échantillon de sang $(3 \mathrm{ml})$ permettant d'obtenir la glycémie de base. Puis après l'ingestion des boissons, des échantillons de sang $(3 \mathrm{ml})$ ont été prélevés via le cathéter dans des tubes contenant du fluorure de sodium oxalate toutes les 15 minutes pendant la première heure puis toutes les 30 minutes à partir de la deuxième heure. Chaque groupe de sujets a eu quatre séances espacées d'une semaine. La première séance a été consacrée à la prise de $100 \mathrm{~g}$ de pain de blé tartiné de margarine accompagnée de 250 $\mathrm{ml}$ d'eau plate pendant 10 minutes. La deuxième séance a été identique à la première selon les recommandations de la FAO/WHO (1998). La troisième séance a été consacrée à la prise des différentes boissons étudiées accompagnée de 100 grammes de pain de blé tartiné de margarine en 10 minutes selon les modalités suivantes : $1000 \mathrm{KJ}$ de sodabi titré à $40^{\circ}$ correspondant à $100 \mathrm{ml}$ de boisson pour l'étude $1 ; 1000 \mathrm{KJ}$ de tchoukoutou titré à $3^{\circ}$ correspondant à $1500 \mathrm{ml}$ de boisson pour l'étude 2; $1000 \mathrm{KJ}$ de vin de palme fermenté titré à $8^{\circ}$ correspondant à $500 \mathrm{ml}$ de boisson pour l'étude $3 ; 1000 \mathrm{KJ}$ de vin de palme frais titré à $3^{\circ}$ correspondant à $1500 \mathrm{ml}$ de boisson pour l'étude 4. La quatrième séance a été consacrée à la prise des mêmes quantités des boissons étudiées consommées à jeun en dix (10) minutes.

\section{Dosage de la glycémie}

Les échantillons de sang prélevés ont été centrifugés à $4000 \mathrm{rpm}$ pendant 15 minutes pour obtenir les plasmas. Le glucose a été dosé dans les plasmas ainsi obtenus par la méthode enzymatique en point final à la glucose oxydase-peroxydase en utilisant les kits du laboratoire Linear Chemicals. Après avoir prélevé $1000 \mu \mathrm{l}$ de réactif (glucose kit) dans des tubes à hémolyse dont le nombre est égal au nombre d'échantillons à doser plus trois autres tubes pour le blanc réactif, l'étalon et le sérum de contrôle, nous avons d'abord ajouté dans le tube à hémolyse correspondant $10 \mu \mathrm{l}$ de plasma de chaque échantillon. Ensuite les tubes à hémolyse ainsi préparés ont été incubés au bain marie à $37^{\circ} \mathrm{C}$ pendant 10 minutes. Enfin la concentration en 
glucose de chaque échantillon a été obtenue à la lecture au spectrophotomètre à $500 \mathrm{~nm}$ après vérification d'une bonne calibration par des lectures successives du blanc réactif, de l'étalon et du sérum de contrôle.

\section{Le tracé des courbes des réponses glycémiques et des diagrammes des aires sous la courbe incrémentale}

Les courbes des réponses glycémiques ont été obtenues à partir des moyennes des glycémies de chaque temps des dix sujets sur chaque étude. Le calcul de la réponse glycémique a été réalisé par la méthode des trapèzes qui détermine l'aire sous la courbe incrémentale (Brouns et al., 2005). Les diagrammes des aires sous la courbe incrémentale ont été obtenus à partir des moyennes des aires sous la courbe calculées par individu au niveau de chaque étude associées à leur déviation standard. L'index glycémique de chaque boisson a été calculé à partir des moyennes des aires sous la courbe incrémentale (Wolever, 1992 ; Wolever et al., 2003 ; John et al., 2008). La charge glycémique $(C G)$ a été obtenue par la formule suivante : $\quad C^{\prime} G=g * \frac{I G}{100} \quad$ où $g$ correspond aux grammes de glucides disponibles consommés et $I G$ est l'index glycémique de l'aliment (Wolever, 1992; Granfeldt et al., 1995; John et al., 2008).

Analyse statistique

Les données ont été analysées à l'aide du logiciel Excel de Microsoft Office 2007. La comparaison des données a été faite par le test du chi carré pour les proportions, l'analyse de la variance (ANOVA) affinée par le LSD test de DUNCAN et le test $t$ de Student pour les moyennes des aires sous la courbe incrémentale calculées. Pour les échantillons dont le test d'homogénéité de variance n'était pas significatif, nous avons utilisé le test de Welche. La différence était significative si $\mathrm{p}<0,05$.

\section{RESULTATS}

La Figure 1 représente l'évolution des moyennes ( \pm écarts-types) de la glycémie dans le temps après la prise du Sodabi à jeun, du pain + sodabi et du pain + eau (étude 1). Le pain + sodabi a réduit la glycémie postprandiale de $20,65 \%$ tandis que la consommation de sodabi à jeun l'a réduit de 81,65\%. La Figure 2 représente l'évolution des moyennes ( \pm écarts-types) de la glycémie dans le temps après la prise du tchoukoutou à jeun, du pain + tchoukoutou et du pain + eau (étude 2). La consommation de pain + tchoukoutou a réduit la glycémie post prandiale de $16,92 \%$ tandis que la consommation de tchoukoutou à jeun l'a réduit de 79,4\%. La Figure 3 représente l'évolution des moyennes ( \pm écarts-types) de la glycémie dans le temps après la prise du vin de palme fermenté à jeun, du pain + vin fermenté et du pain + eau (étude 3). La consommation du pain + vin fermenté a réduit la glycémie post prandiale de $26,87 \%$ tandis que la consommation du vin de palme fermenté à jeun l'a réduit de 95,72\%. La Figure 4 représente l'évolution des moyennes ( \pm écarts-types) de la glycémie dans le temps après la prise du vin de palme frais à jeun, du pain + vin de palme frais et du pain + eau (étude 4). La consommation de vin de palme frais associé à 100 grammes de pain a réduit la glycémie post prandiale de $64,06 \%$ tandis que la consommation de vin de palme frais à jeun a induit une diminution de la réponse glycémique de $88,33 \%$.

Le Tableau 3 montre les valeurs moyennes des aires sous la courbe incrémentale des quatre études. Les différentes moyennes des aires sous la courbe incrémentale des quatre boissons alcoolisées consommées à jeun par sexe bien que variable selon le sexe n'ont pas été significativement différentes à $5 \%(\mathrm{p}=0,41,0,52,0,44$ et 0,45 respectivement pour la consommation de sodabi, de vin de palme fermenté, de tchoukoutou et de vin de palme frais).

Les index et charge glycémiques des quatre boissons consommées à jeun figurent dans le Tableau 4. 
Tableau 1: Caractéristiques physico-chimiques des boissons utilisées pour $100 \mathrm{~cm}^{3}$ (Chevassus et Favier, 1976 ; Kayode et al., 2007 ; Adeleke et Abiodun, 2010).

\begin{tabular}{lcccccccc}
\hline Boissons & $\begin{array}{l}\text { Eau } \\
(\boldsymbol{\%})\end{array}$ & $\begin{array}{c}\text { Protides } \\
(\mathbf{g})\end{array}$ & $\begin{array}{c}\text { Lipides } \\
(\mathbf{g})\end{array}$ & $\begin{array}{c}\text { Glucides } \\
(\mathbf{g})\end{array}$ & $\begin{array}{c}\mathbf{C a}^{++} \\
(\mathbf{m g})\end{array}$ & Calories & $\mathbf{p H}$ & $\begin{array}{c}\text { Ethanol } \\
(\boldsymbol{\%})\end{array}$ \\
\hline $\begin{array}{l}\text { Tchoukoutou } \\
\text { Vin de palme } \\
\text { frais }\end{array}$ & 96,4 & 0,3 & - & 33,4 & 1,4 & 35 & 3,2 & 3,03 \\
$\begin{array}{l}\text { Vin de palme } \\
\text { fermenté }\end{array}$ & 99,7 & 0,2 & - & 99 & 2,5 & 40 & 3,1 & 3,1 \\
Sodabi & 93,3 & 0,4 & - & 1 & 2,5 & 2 & 3 & 7,6 \\
\hline
\end{tabular}

Tableau 2 : Paramètres des sujets sélectionnés.

\begin{tabular}{lcc}
\hline & Valeurs obtenues & Valeurs de référence \\
\hline $\mathbf{n}$ & 40 & \\
Hommes / Femmes & $24 / 16$ & \\
Âge en année & $24,10 \pm 2,22$ & \\
Pression artérielle systolique (mmHg) & $12,64 \pm 0,9$ & $<130$ \\
Pression artérielle diastolique $(\mathbf{m m H g})$ & $84,3 \pm 0,6$ & $<85$ \\
Glycémie à jeun (mmol/l) & $4,11 \pm 0,48$ & $3,89-6,11$ \\
Urée sanguine (mmol/l) & $3,08 \pm 0,48$ & $2,49-9,13$ \\
\hline & $+97,65 \pm 4,39$ & $61,09-123$ \\
Créatinine plasmatique & $\uparrow 72,88 \pm 3,25$ & $53-106$ \\
\hline ALAT & $28,01 \pm 10,13$ & $<48$ \\
ASAT & $19,08 \pm 7,49$ & $<48$ \\
\hline
\end{tabular}

Tableau 3 : Valeurs moyennes des aires sous la courbe incrémentale des quatre études.

\begin{tabular}{|c|c|c|}
\hline & & $\begin{array}{l}\text { Aire sous la courbe incrémentale en } \\
\text { mmol/L.min (moyenne } \pm \text { écart-type) }\end{array}$ \\
\hline \multirow{4}{*}{$\begin{array}{l}\text { Etude } 1 \\
(\mathbf{n}=\mathbf{1 0})\end{array}$} & Pain + eau & $86,32 \pm 17,74$ \\
\hline & Sodabi +pain & $68,80 \pm 17,62$ \\
\hline & Sodabi à jeun & $15,91 \pm 7,65$ \\
\hline & Pain +eau & $116,76 \pm 16,32$ \\
\hline \multirow{3}{*}{$\begin{array}{l}\text { Etude } 2 \\
(\mathbf{n}=\mathbf{1 0})\end{array}$} & Tchoukoutou + Pain & $95,93 \pm 13,86$ \\
\hline & Tchoukoutou à jeun & $23,78 \pm 6,66$ \\
\hline & Pain +eau & $124,57 \pm 14,16$ \\
\hline \multirow{3}{*}{$\begin{array}{l}\text { Etude } 3 \\
(\mathbf{n}=\mathbf{1 0})\end{array}$} & Vin de palme fermenté + pain & $89,24 \pm 12,62$ \\
\hline & Vin de palme fermenté à jeun & $5,22 \pm 3,45$ \\
\hline & Pain + eau & $88,21 \pm 14,61$ \\
\hline Etude 4 & Vin de palme frais + pain & $31,33 \pm 10,93$ \\
\hline$(\mathbf{n}=10)$ & Vin de palme frais à jeun & $10,17 \pm 5,62$ \\
\hline
\end{tabular}


Tableau 4 : Index et charge glycémiques des différentes boissons étudiées à jeun.

\begin{tabular}{lccc}
\hline Boissons & $\begin{array}{c}\text { Aire sous la courbe incrémentale } \\
\text { (mmol/L.min) }\end{array}$ & $\begin{array}{c}\text { Index } \\
\text { glycémique (\%) }\end{array}$ & $\begin{array}{c}\text { Charge } \\
\text { glycémique (\%) }\end{array}$ \\
\hline Sodabi & 25,91 & 18,34 & NP \\
Tchoukoutou & 23,78 & 20,59 & 68,77 \\
Vin de palme & 5,22 & 4,27 & 0,42 \\
fermenté & & & \\
Vin de palme frais & 10,17 & 11,66 & 115,43 \\
\hline \multicolumn{2}{c}{$\mathrm{NP}=$ non précisé }
\end{tabular}

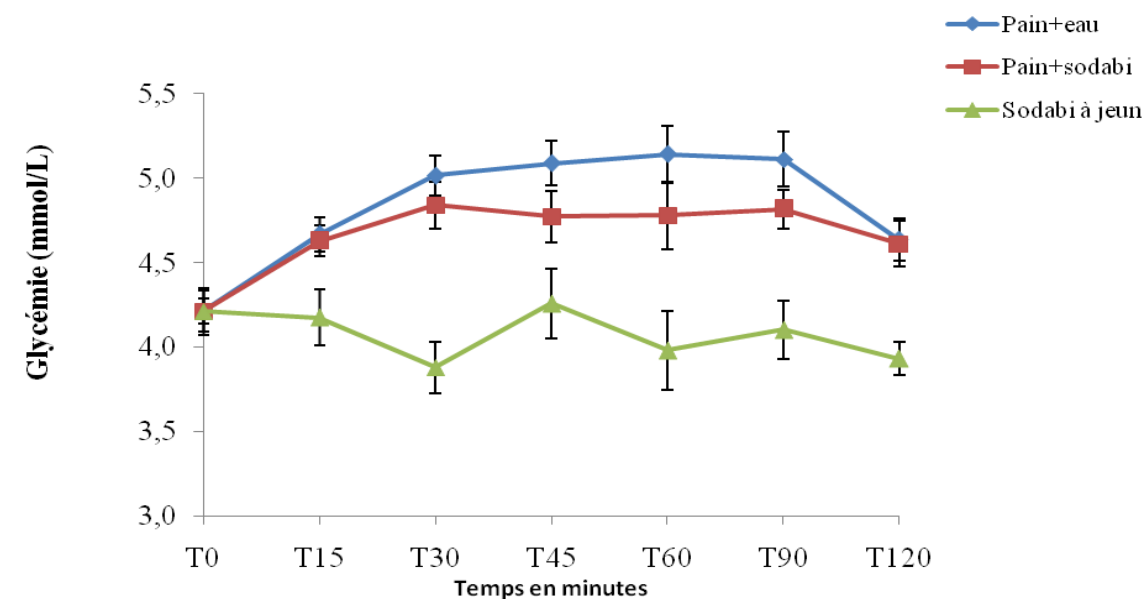

Figure 1: Moyennes ( \pm écarts-types) des variations de la glycémie plasmatique en fonction du temps de l'étude 1.

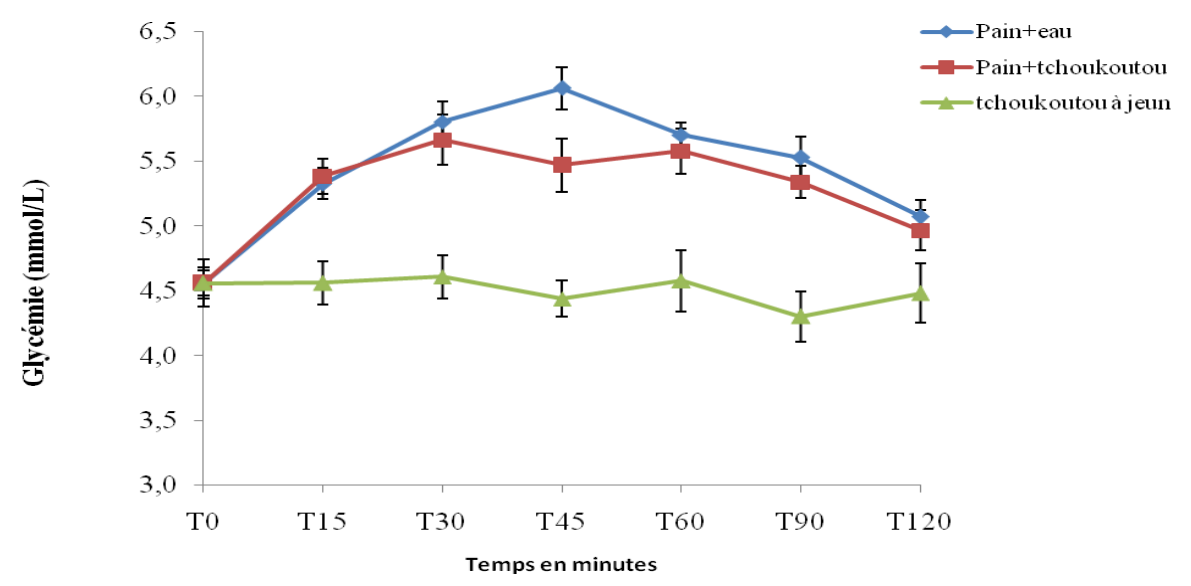

Figure 2: Moyennes ( \pm écarts-types) des variations de la glycémie plasmatique en fonction du temps de l'étude 2. 


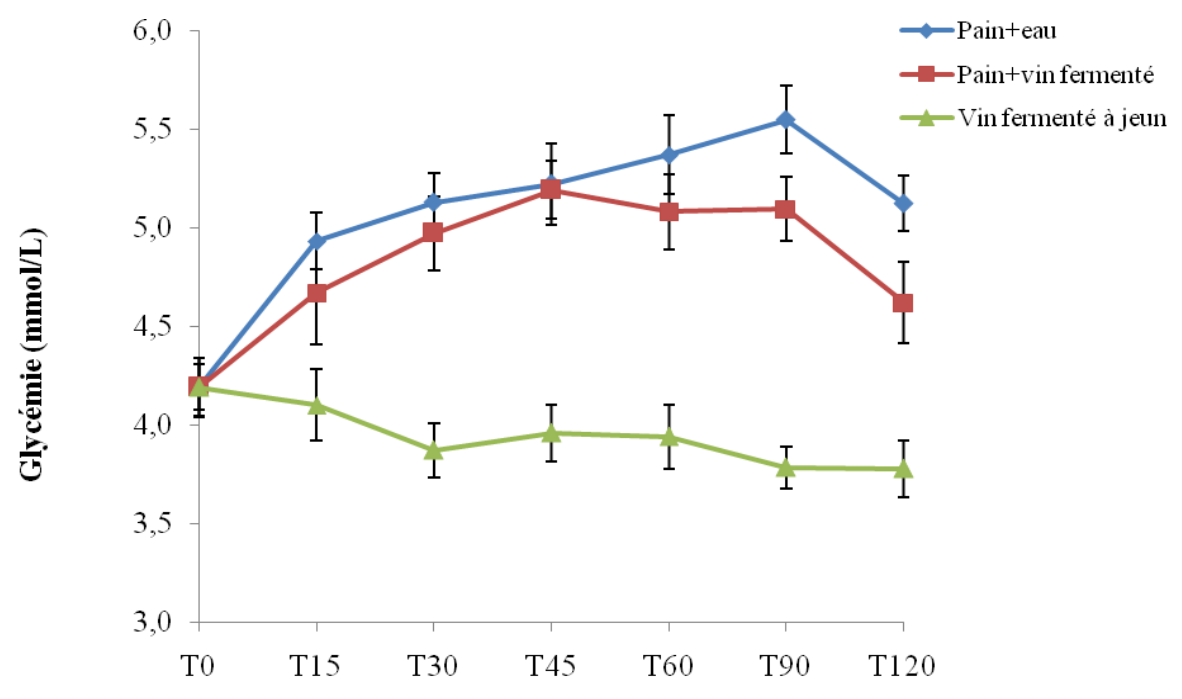

Temps en minutes

Figure 3 : Moyennes ( \pm écarts-types) des variations de la glycémie plasmatique en fonction du temps de l'étude 3.

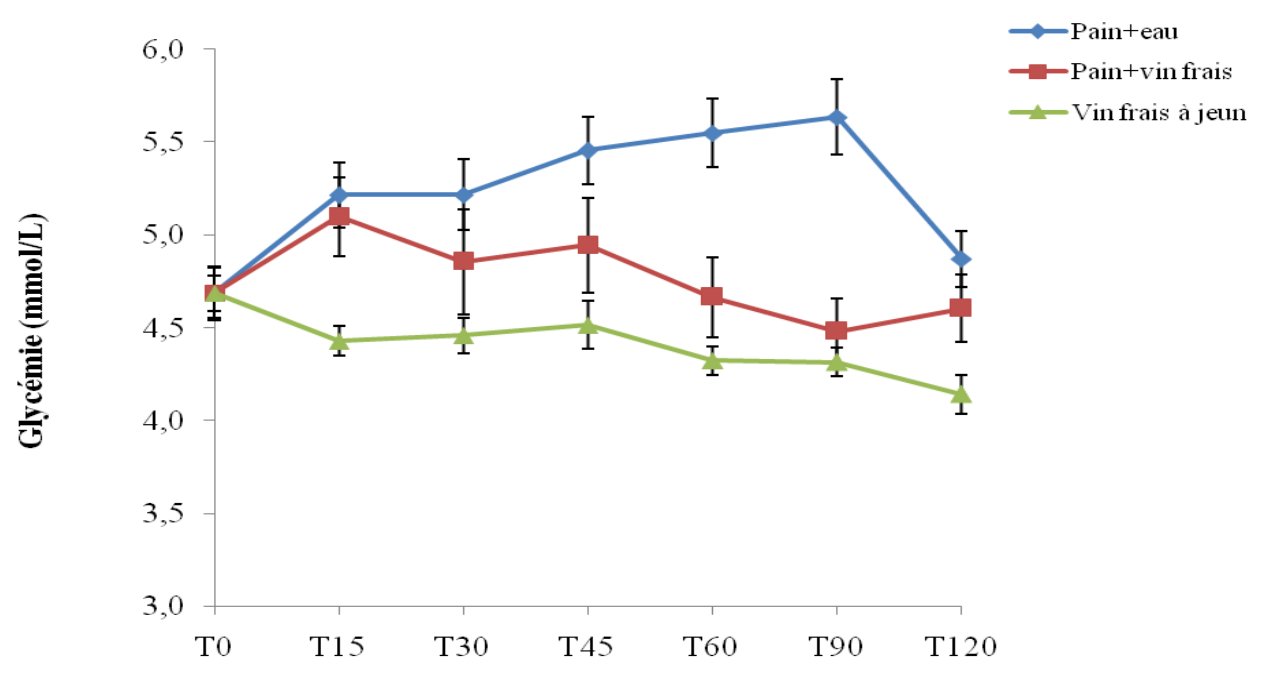

Temps en minutes

Figure 4 : Moyennes ( \pm écarts-types) des variations de la glycémie plasmatique en fonction du temps de l'étude 4. 


\section{DISCUSSION}

Ce travail nous a permis d'explorer, par une étude expérimentale, la réponse glycémique induite par quatre boissons alcoolisées de fabrication locale du Bénin chez des sujets adultes jeunes et sains. Les quatre boissons testées seules ou accompagnées de pain ont réduit la glycémie post prandiale à des degrés divers.

Cependant malgré la satisfaction aux critères de validité d'une bonne étude sur les réponses glycémiques, ce travail a souffert de diverses insuffisances: l'utilisation du pain conventionnel à la place du pain blanc non disponible sur la marché local, l'utilisation du sang veineux en l'absence d'un automate pour le traitement de sang capillaire et l'utilisation des résultats de plusieurs travaux réalisés sur les compositions des différentes boissons étudiées (Chevassus et Favier, 1976 ; Kayode et al., 2006; Adeleke et Abiodun, 2010) en l'absence d'équipements biotechnologiques dans notre laboratoire pour les déterminer. Ces insuffisances ne remettent pas en cause les conclusions de notre étude vu les faibles variations des valeurs des glycémies obtenues lors de la réalisation des deux essais sur l'aliment de base et l'allure générale des courbes obtenues, et, les procédés de fabrication de ces boissons alcoolisées locales identiques dans toute l'Afrique de l'Ouest. De plus, certaines études comparatives entre la glycémie capillaire et la glycémie veineuse préconisent l'utilisation de la glycémie veineuse pour confirmer les valeurs de la glycémie capillaire surtout lorsqu'il existe des valeurs capillaires intermédiaires (LarssonCohn, 1976).

\section{Boissons consommées à jeun}

Les valeurs moyennes des aires sous la courbe incrémentale obtenues dans notre étude pour le sodabi $(15,91 \pm 7,65)$ et le vin de palme frais $(10,17 \pm 5,62)$, bien que plus élevées que celles rapportées par Brand-Miller et al. (2007) en Australie qui étaient de $10 \pm 5$ pour le Gin et de $7 \pm 3$ pour le vin, ne sont pas statistiquement différentes (respectivement $\mathrm{p}=0,054$ et 0,14 ). Par contre, la moyenne de l'aire sous la courbe incrémentale du tchoukoutou $(23,78 \pm 6,66)$ est inférieure à celle retrouvée par les mêmes auteurs pour la consommation de la bière qui était de $58 \pm 11(\mathrm{p}<0,001)$. La moyenne de l'aire sous la courbe rapportée par BrandMiller et al. (2007) pour le vin blanc $(7 \pm 3)$ est supérieure à celle obtenue au cours de notre étude pour le vin de palme fermenté $(5,22 \pm 3,45)(\mathrm{p}<0,001)$.

Plusieurs raisons peuvent expliquer ces différents résultats. La quantité d'alcool dans le sodabi ( $33,6 \mathrm{~g}$ pour $100 \mathrm{ml}$ de boisson) est inférieure à celle du Gin $(47 \mathrm{~g}$ pour $100 \mathrm{ml}$ de boisson); de plus, la présence d'acétaldéhyde dans le sodabi lié à un mauvais processus de fermentation pourrait atténuer la baisse de la réponse glycémique (Obiora et al., 2007). La présence de petites quantités d'hydrates de carbones (13 g pour $100 \mathrm{ml}$ de boisson) rapidement digérables dans la bière industrielle (Brand-Miller et al., 2007) pourrait contribuer potentiellement à augmenter la réponse glycémique de manière significative par rapport au tchoukoutou qui n'en contient que 4 grammes (Chevassus et Favier, 1976). En outre, le processus fondamental de la fabrication de la bière traditionnelle en Afrique de l'Ouest est caractérisé par le maltage où l'amidon est transformé en sucre simple puis en acide acétique (Ibegbulem et al., 2003); ce processus d'acidification du produit serait favorable à une baisse importante de la glycémie (Östman et al., 2005). Les résultats obtenus avec le vin de palme frais peuvent s'expliquer par la prédominance du saccharose au détriment du glucose dans cette forme de vin de palme (Noll, 2008). De plus, 
la forte charge glycémique du vin de palme frais $(115,43 \%)$ par rapport au vin blanc $(7 \%)$ (Brand-Miller et al., 2007) pourrait expliquer ce résultat. Avec le vin de palme fermenté, les résultats obtenus peuvent être dus à une différence dans l'origine du vin. En effet, le vin de palme local utilisé dans notre étude a été produit à partir de la sève d'Elaeis guineensis qui est une plante typiquement tropicale (Yavo et al., 2002) tandis que le vin industrialisé utilisé dans l'étude de BrandMiller et al. (2007) provient de la fermentation de fruits divers. Aussi la présence de composés phénoliques (Obiora et al., 2007) dans le vin de palme en général pourrait-elle expliquer la diminution progressive de la réponse glycémique observée car la littérature rapporte que ces composés phénoliques diminuent l'absorption intestinale de glucose et stimulent fortement la sécrétion d'insuline (Anderson et Polansky, 2002).

\section{Boissons consommées avec 100 grammes de pain}

Les moyennes des aires sous la courbe incrémentale du tchoukoutou $(95,93 \pm 13,86)$, du vin de palme fermenté $(89,24 \pm 12,62)$ et du sodabi $(68,80 \pm 17,62)$ bien que différentes de celles rapportées par Brand-Miller et al. (2007) en Australie qui étaient de $84 \pm 11$ pour la bière $(\mathrm{p}=0,30)$, de $63 \pm 6$ pour le vin $(\mathrm{p}=0,05)$ et de $80 \pm 12$ pour le Gin $(\mathrm{p}=0,45)$ n'étaient pas significatives. Par contre, la moyenne de l'aire sous la courbe incrémentale du vin de palme frais de notre étude $(31,33 \pm$ $10,93)$ était inférieure à celle retrouvée par les mêmes auteurs $(63 \pm 6)$ en ce qui concerne la consommation de vin $(p<0,001)$.

Ces résultats peuvent s'expliquer par différents faits. Malgré une quantité presque égale d'alcool contenue dans le tchoukoutou (33,6 g pour $100 \mathrm{ml}$ de boisson) (Adeleke et Abiodun, 2010) et dans la bière industrielle
(34 g pour $100 \mathrm{ml}$ de boisson) (Brand-Miller et al., 2007), l'index glycémique de la bière industrielle $(5,8 \%)$ utilisée par Brand-Miller et al. (2007) est inférieur à celui du tchoukoutou $(20,59 \%)$ favorisant ainsi une meilleure stimulation de la glycémie post prandiale. Le vin de palme fermenté contient environ $20 \mathrm{~g}$ d'alcool pour $100 \mathrm{ml}$ de boisson comparativement au vin blanc qui en contient 39 g. Aussi le taux élevé de protéines dans le vin blanc (Brand-Miller et al., 2007) par rapport au vin de palme fermenté (Adeleke et Abiodun, 2010) pourrait-il expliquer cette modification de la réponse glycémique. En effet, les acides aminés dominants dans les protéines d'origine végétale (en particulier la leucine) sont très insulinotropes (Layman, 2003). Le vin de palme frais qui possède la charge glycémique la plus élevée aurait probablement entrainé «un syndrome hypoglycémique postprandial idiopathique » (Vialettes et Silvestre, 1993).

\section{Comparaison des effets des différentes boissons}

Les résultats obtenus dans notre travail ont montré que chaque boisson influençait différemment la réponse glycémique en fonction de sa composition biochimique comme l'ont signalé plusieurs auteurs. Östman et al. (2001) en Suède ont corroboré nos résultats sur le vin de palme fermenté en montrant que la forte teneur en acide acétique $\mathrm{du}$ lait favoriserait son fort pouvoir hypoglycémique. Vuksan et al. (2001) au Canada puis Bryans et al. (2007) à Londres ont corroboré nos résultats sur le vin de palme en général en montrant que la présence de composés phénoliques dans le thé noir et le ginseng (Panax quinquefolius L) entraine une forte hypoglycémie. Brand-Miller et al. (2007) à Sydney ont montré que la bière industrielle réduisait plus la glycémie que le Gin et le vin à jeun, ce qui est en contradiction 
avec nos résultats. En effet, dans notre travail, les vins de palme étudiés (fermenté et frais) ont réduit plus la glycémie que le tchoukoutou et le sodabi. Ceci pourrait s'expliquer non seulement par le caractère acide du vin industriel qui favoriserait le ralentissement de la vidange gastrique, mais aussi par la présence d'amylase dans le vin qui a la particularité d'inhiber la glucosidase (Liljeberg et al., 1995).

\section{Effets des boissons en fonction du sexe}

Dans notre étude, la réponse glycémique variait en fonction du sexe mais de façon non significative. Brand-Miller et al. (2007) ont montré que la réduction de la glycémie chez les hommes était plus importante que chez les femmes pour la consommation de vin blanc. Nous avons abouti au même résultat en ce qui concerne la consommation de vin de palme frais. S'agissant du vin de palme fermenté, notre étude a cependant montré que la réduction de la glycémie était beaucoup plus marquée chez les femmes. Quant au tchoukoutou, il réduit plus la glycémie chez les hommes tandis que le sodabi réduit plus la glycémie chez les femmes. Les études réalisées sur des rats ont pu montrer qu'il existait une différence de réponse glycémique entre les deux sexes en spécifiant que les rats de sexe féminin étaient plus disposés à avoir une hypoglycémie que ceux de sexe masculin, même si chez les humains cette différence reste à démontrer (Sumida et al., 2007).

\section{Aspects nutritionnels}

L'intérêt des aliments à index glycémique faible ou modéré a été évoqué depuis plusieurs années dans le diabète sucré (Foster-Powell et al., 2002). Des réponses glycémiques modérées en postprandiale sont en effet souhaitables pour une bonne homéostasie $\mathrm{du}$ fonctionnement humain.
Toutes nos boissons étudiées avaient un index glycémique bas sans toute fois entrainer une hypoglycémie réelle (< $3 \mathrm{mmol} / \mathrm{L})$. Ces résultats ont été corroborés par Brand-Miller et al. (2007) sauf au niveau de la bière industrielle où ils ont noté un index glycémique modéré de $54 \%$. Cependant le vin de palme frais possédait la charge glycémique la plus élevée $(115,43 \%)$. Ainsi toutes les boissons étudiées doivent être proscrites chez les sujets diabétiques de type 1 mais pourront être consommées avec modération chez les sujets diabétiques de type 2 en dehors du vin de palme frais.

Cette étude a montré que les boissons alcoolisées locales du Bénin influencent différemment la réponse glycémique chez des sujets adultes jeunes et sains. Elle permet donc d'envisager leur utilisation dans la prise en charge diététique du diabète sucré de type 2.

\section{REFERENCES}

Adeleke RO, Abiodun OA. 2010. Physicochemical Properties of Commercial Local Beverages in Osun State, Nigeria. Pakistan Journal of Nutrition, 9(9): 853855.

Anderson RA, Polansky MM. 2002. Tea enhances insulin activity. J. Agric. Food Chem., 50: 7182-7186.

Brand-Miller J, Fatema K, Middlemiss C, Bare M, Liu V, Atkinson F, Petocz P. 2007. Effect of alcoholic beverages on postprandial glycémia and insulinemia in lean, young, healthy adults. Am. J. Clin. Nutr., 85: 1545-1551.

Brouns F, Bjorck I, Frayn KN, Gibbs AL, Lang V, Slama G, Wolever TM. 2005. Glycemic index methodology. Nutr. Res. Rev., 18: 145-171.

Bryans JA, Judd PA, Ellis PR. 2007. The Effect of Consuming Instant Black Tea on Postprandial Plasma Glucose and 
Insulin Concentrations in Healthy Humans. Journal of the American College of Nutrition, 26: 471-477.

Chevassus A, Favier JC. 1976. Technologie traditionnelle et valeur nutritive des bières de sorgho du Cameroun. Cahier de Nutrition et de Diététique, 11(2): 89-104.

FAO/WHO expert consultation. 1998. Carbohydrates in human nutrition: report of a joint FAO/WHO Expert Consultation, Rome, 14-18 April, 1997. Rome: Food and Agriculture Organization. (FAO Food and Nutrition paper 66).

Foster-Powell K, Holt HAS and Brand-Miller JC. 2002. International table of glycemic index and glycemic load values. Am. J. Clin. Nutr., 76: 5-56.

Granfeldt Y, Hagander B, Bjorck I. 1995. Metabolic responses to starch in oat and wheat products. On the importance of food structure, incomplete gelatinization or presence of viscous dietary fibre. Eur. J. Clin. Nutr., 49: 189-199.

Hunt JN, Knox MT. 1972. The slowing of gastric emptying by four strong acids and three weak acids. J. Physiol., 222: 187208.

Ibegbulem CO, Alisi CS, Anyaegbu OM. 2003. Influence of Storage Conditions on the Quality Attributes of Kunu and Burukut. Journal of Agriculture and Food Science, 1(2): 107-113.

John A, Monr J, Shaw M. 2008. Glycemic impact, glycemic glucose equivalents, glycemic index, and glycemic load: definitions, distinctions, and implications. Am. J. Clin. Nutr., 87: 237-243.

Kayode P, Hounhouigan J, Martinus J. 2007. Household production of sorghum beer in Benin: technological and socio-economic aspects. International Journal of Consumer Studies, 31: 258-264.
Larsson-Cohn U. 1976. Differences between capillary and venous blood glucose duringoral glucose tolerance tests. Scand. J. Clin. Lab. Invest., 36: 805-808.

Layman DK. 2003. The role of leucine in weight loss diets and glucose homeostasis. J. Nutr., 133(1): 261S-27S.

Liljeberg HGM, Lönner $\mathrm{CH}$, Björck IME. 1995. Sourdough fermentation or addition of organic acids or corresponding salts to bread improves nutritional properties of starch in healthy humans. J. Nutr., 125: 1503-1511.

Noll RG. 2008. The Wines of West Africa: History, Technology and tasting notes. Journal of Wine Economics, 3: 85-94.

Obiora SE, Brands B, Jürgen R, Lachenmeier DW. 2007. Composition of surrogate alcohol from south-eastern Nigeria. African Journal of Drug and Alcohol Studies, 6(2): 64-67.

Östman EM, Liljeberg H, Björck IME. 2001. Inconsistency between Glycemic and insulinemia responses to regular and fermented milk products. Am. J. Clin. Nutr., 74: 96-100.

Östman E, Granfeldt Y, Persson L, Bjorck I. 2005. Vinegar supplementation lowers glucose and insulin responses and increases satiety after a bread meal in healthy subjects. Eur. J. Clin. Nutr., 59: 983-988.

Sporer KA, Ernst AA, Conte A. 1992. The incidence of ethanol-induced hypoglycemia. Am. J. Emerg. Med., 10: 403-405.

Sumida KD, Hill JM, Matveyenko AV. 2007. Sex Differences in Hepatic Gluconeogenic Capacity after Chronic Alcohol Consumption. Clinical Medicine Research, 5(3): 193-202.

Todesco T, Rao AV, Bosello O, Jenkins DJA. 1991. Propionate lowers blood glucose 
and alters lipid metabolism in healthy subjects. Am. J. Clin. Nutr., 54: 860-865.

Verdy M, Diallo G. 1968. Hypoglycémie et alcool. Canad. Med. Ass. J., 98: 827-830.

Vialettes B, Silvestre P. 1993. Les Hypoglycémies fonctionnelles. Cah. Nutr. Diet., 28: 173-175.

Vuksan V, Sievenpiper JL, Wong J, Xu Z, Beljan-Zdravkovic U, Arnason JT, Assinewe V, Stavro MP, Jenkins AL, Leiter LA, Thomas Francis T. 2001. American ginseng (Panax quinquefolius L) attenuates postprandial glycemia in a time-dependent but not dose-dependent manner in healthy individuals. Am. J. Clin. Nutr., 73: 753-758.

Wolever TMS. 1992. Glycemic index vs glycemic response: non-synonymous terms. Diabetes Care, 15: 1436- 1437.

Wolever TM, Vorster HH, Bjorck I. 2003. Determination of the glycaemic index of foods: interlaboratory study. Eur. J. Clin. Nutr., 57: 475-482.

Yavo E, Mollet M, Girardin O, Sorg J P, Herzog F. 2002. Le vin de palme: aliment et source de revenu pour les populations rurales en Cote d'Ivoire. Schweiz Z. Forstwes, 153(4): 123-129. 